\title{
APPROXIMATION OF ANALYTIC MULTIFUNCTIONS
}

\author{
ZBIGNIEW SLODKOWSKI
}

(Communicated by Paul S. Muhly)

\begin{abstract}
Set-valued generalizations of analytic functions are defined by a form of local maximum principle. It is shown that they are identical with limits of decreasing sequences of multifunctions whose graphs are locally covered by graphs of single-valued analytic mappings.
\end{abstract}

\section{INTRODUCTION}

It is a natural question how to generalize the notion of an analytic function to the context of set-valued functions $z \rightarrow K(z): G \rightarrow 2^{\mathrm{C}^{n}}$, where $G \subset \mathbb{C}^{k}$. In case $k=n=1$, Oka [3] defined analytic set-valued functions (called here analytic multifunctions) by requiring that the complement of the graph of $K$ in $G \times \mathbb{C}$ is pseudoconvex. Oka's definition does not have an obvious extension to the higher-dimensional case. The author proposed two generalizations, first in [4, Definition 5.1], and then a more restrictive one (and in case $k>1$ actually different) in [6, §2], [9, Definition 0.2]. The existence of two definitions may raise some doubt whether any of them is the proper one. We show in this paper that analytic multifunctions in the latter sense (cf. Definition 2.1 below) can be approximated by a decreasing sequence of multifunctions which are locally union of graphs of single-valued analytic mappings (Theorem 1.3).

In addition to confirming the second definition, this result gives an insight into the structure of analytic multifunctions and allows for transparent explanation of many of their properties, previously available by other methods (see, $\S 6)$.

\section{Results}

Definition 1.1 [6, 9]. An usc compact-valued function $z \rightarrow K(z): G \rightarrow 2^{\mathrm{C}^{n}}$, $G \subset \mathbb{C}^{k}$, is called analytic if for every hyperplane $L \subset \mathbb{C}^{k+n}$ with $\operatorname{dim}_{\mathrm{C}} L=$ $n+1$, for every polynomial $p(z, w)$ and for every ball $B, \max (\operatorname{Re} p) \mid X \cap L \cap$ $\bar{B} \leq \max (\operatorname{Re} p) \mid X \cap L \cap \partial B$, provided $\bar{B} \cap X$ is compact, where $X=\operatorname{gr}(K)=$ $\{(z, w): z \in G, w \in K(z)\}$.

Received by the editors May 2, 1988 and, in revised form, July 20, 1988.

1980 Mathematics Subject Classification (1985 Revision). Primary 32F05, 32F15; Secondary 32E20.

Key words and phrases. $q$-pseudoconvex sets, $q$-plurisubharmonic functions, analytic multifunctions, polynomial hulls.

This research was supported in part by the National Science Foundation grant DMS-870227. 
Definition 1.2(a). We say that $K: G \rightarrow 2^{\mathrm{C}^{n}}$ is a trivial analytic multifunction if for every $z_{0} \in G, w_{0} \in K\left(z_{0}\right)$, there is an analytic mapping $f: G \rightarrow \mathbb{C}^{n}$, such that $f\left(z_{0}\right)=w_{0}$ and $f(z) \in K(z)$ for $z \in G$.

(b) We say that $K: G \rightarrow 2^{\mathrm{C}^{n}}$ is a locally trivial analytic multifunction if there is an open covering $\left\{G_{t}\right\}$ of $G$ such that $K \mid G_{t}$ is a trivial analytic multifunction for every $G_{t}$.

We can formulate now the main result of this paper.

Theorem 1.3. If $z \rightarrow K(z): G \rightarrow 2^{\mathbb{C}^{n}}, G \subset \mathbb{C}^{k}$, is an analytic multifunction, then there exists a countable covering $\left\{G_{s}\right\}_{s=1}$ of $G$, such that $G_{s} \subset G_{s+1}, s=$ $1,2, \ldots$, and a sequence of locally trivial analytic multifunctions $K_{s}: G_{s} \rightarrow 2^{\mathrm{C}^{n}}$, such that $K_{r+1}(z) \subset K_{r}(z)$ for $z \in G_{s}$ and $r \geq s$, and $\bigcap_{r \geq s} K_{r}(z)=K(z)$, $z \in G_{s}$.

Remark 1.4. The last theorem can be improved if the multifunction $K(\cdot)$ is uniformly bounded (i.e., $\sup \{|w|: z \in G, w \in K(z)\}<+\infty$ ) and continuous (with respect to the Hausdorff topology in the space of all nonempty compact subsets of $\mathbb{C}^{n}$ ). Then, the conclusions of the last theorem are valid with $G_{s}=G$ for all $s$.

It is convenient to use the following terminology.

Terminology 1.5. We will say that a multifunction $K: G \rightarrow 2^{\mathrm{C}^{n}}$ has property (MA) on $G$, if it admits a sequence of locally trivial analytic multifunctions $K_{s}: G \rightarrow 2^{\mathrm{C}^{n}}$ such that $K_{s+1}(z) \subset K_{s}(z)$ and $\bigcap_{s} K_{s}(z)=K(z)$ for $z \in G$, $s=1,2, \ldots$.

We will prove Theorem 1.3 in $\S 5$, after presenting, in $\S \S 2-4$, auxiliary results on the (MA)-property and $q$-plurisubharmonic functions.

\section{APPLICATION OF POLYNOMIAL hULLS}

Lemma 2.1. Given $\varepsilon>0$ and $0<r<\varepsilon^{2}$, let

$$
X=\{(\zeta, w):|\zeta|=r, \operatorname{dist}(w,\{\sqrt{\zeta},-\sqrt{\zeta}\})<\varepsilon\} .
$$

Denote by $Y$ the polynomial hull of $X$ and by $Y(z),|z| \leq r$, the section $Y(z)=\{w:(z, w) \in Y\}$. Then $Y(0)$ is a circle with radius $R=R(\varepsilon, r)$ bigger than $\varepsilon$. Furthermore,

$$
\{(0, w):|w|<R\} \subset \operatorname{Int}(Y) .
$$

Corollary 2.2. Denote $L_{0}(z)=\{\sqrt{z},-\sqrt{z}\}, z \in \mathbb{C}$. Then, the multifunction $z \rightarrow L_{0}(z): \mathbb{C} \rightarrow 2^{\mathbb{C}}$ has property $(M A)$.

Proof of Lemma 2.1. Since $X$ is invariant with respect to the group of linear transformations $(z, w) \rightarrow\left(\alpha^{2} z, \alpha w\right): \mathbb{C}^{2} \rightarrow \mathbb{C}^{2}$, where $|\alpha|=1$, so is $Y$. Thus 
$Y(0)$ is preserved by rotations and, being polynomially convex, is a circle. Note, further, that the set $Z=\{(z, w):|z|<r$, $\operatorname{dist}(w,\{\sqrt{z},-\sqrt{z}\}) \leq \varepsilon\}$ has the local maximum property relative to polynomials. Since $\bar{Z} \backslash Z \subset X, Y$ contains $Z$. Since $Z(0)$ (=the section of $Z$ at 0$)=D(0, \varepsilon)$, we get $\varepsilon \leq R$. We will show that assuming $R=\varepsilon$ leads to contradiction.

Assertion. The relative boundary of $Y \backslash X$ in $D(0, r) \times \mathbb{C}$ is covered by graphs of bounded analytic functions defined in $D(0, r)$.

If $R=\varepsilon$, then $(0, \varepsilon) \in \partial Y$ and, by the assertion, there is $f \in H^{\infty}(D(0, r))$ such that $f(0)=\varepsilon$ and $(z, f(z)) \in \partial Y$ for $|z|<r$. Since $Y \supset Z$,

$$
|f(z)-\sqrt{z}| \geq \varepsilon, \quad|f(z)+\sqrt{z}| \geq \varepsilon, \quad \text { for }|z|<r .
$$

and $h(z)=f(z)^{2}-z$ satisfies $|h(z)| \geq \varepsilon^{2}=h(0),|z|<r$. Hence $h(z)=$ $f(z)^{2}-z=\varepsilon^{2}$, which together with (2.2) implies $|f(z)-\sqrt{z}|=\varepsilon,|f(z)+\sqrt{z}|=$ $\varepsilon$, and $f(z)=\left(\varepsilon^{2}+z\right)^{1 / 2},|z|<r$. This is a contradiction.

To prove the inclusion $(2.1)$, note first that $D(0, r) \times \bar{D}\left(0, r^{1 / 2}\right) \subset X$. For any analytic function $f$ in $D(0, r)$ such that $\operatorname{gr} f \subset Y$, denote $Z_{f}=\{(z, w)$ : $|z|<r, w \in \operatorname{co}\left(\bar{D}\left(0, r^{1 / 2}\right) \cup\{f(z)\}\right)$, where co=the convex hull. Since $Z_{f}$ has the local maximum property, $Z_{f} \subset Y$. Fix now $R_{1}<R$ and consider $\delta>0$ (to be specified later). Choose a finite $\delta$-net $\zeta_{1}, \ldots, \zeta_{m}$ in $\partial D(0, R)$. By the assertion, there are analytic functions $f_{1}, \ldots, f_{m}$ such that $f_{j}(0)=\zeta_{j}$ and gr $f_{j} \subset Y$ for $j=1, \ldots, m$. One can show easily that if $\delta>0, \eta>0$ are chosen small enough, then $\bigcup_{j} X_{f_{j}}$ contains the set $D(0, \eta) \times D\left(0, R_{1}\right)$. This being true for any $R_{1}<R$, inclusion (2.1) follows.

The assertion follows from the main result of Forstneric [2] by considering a sequence of $C^{(2)}$-smooth manifolds $M_{n} \subset \partial D(0, r) \times \mathbb{C}$ such that for each $|\zeta|=$ $r$, the sections $M_{n}^{\zeta}, n=1,2, \ldots$, are simple closed curves whose polynomial hulls form a decreasing sequence converging to $Y(\zeta)$. By [2, Theorem 3(iii)], the assertion holds for $Y_{n}=$ the polynomial hull of $M_{n}$, and so it holds for $Y$. (Use Montel theorem and $Y=\bigcap_{n} Y_{n}$ ). Q.E.D.

Proof of Corollary 2.2. Fix $\varepsilon>0,0<r<\varepsilon^{2}$ and define $Y$ as in the last lemma, with $Y(0)=\bar{D}(0, R)$. Let $K^{\varepsilon}(z)=\left\{w \in \mathbb{C}: \operatorname{dist}\left(w,\left\{z^{1 / 2},-z^{1 / 2}\right\} \leq \varepsilon\right\}\right.$, $z \in \mathbb{C}$. Choose $\eta \in(\varepsilon / R, 1)$. Let $L^{\varepsilon, r}(z)=K^{\varepsilon}(z)$ for $|z|>r$ and $L^{\varepsilon, r}(z)=$ $K^{\varepsilon}(z) \cup\{\eta w: w \in Y(z)\}$, for $|z| \leq r$. Clearly, $L^{\varepsilon, r}: \mathbb{C} \rightarrow 2^{\mathbb{C}}$ is an usc compactvalued function; we will check that it is a locally trivial analytic multifunction. By (2.1), there is $\delta>0$ such that $\operatorname{gr}\left(L^{\varepsilon, r} \mid D(0, \delta)\right)=Y \cap D(0, \delta) \times \mathbb{C}$, which is covered by graphs of analytic functions by Forstneric [2] (cf. the assertion in the previous proof), and so $L^{\varepsilon, r} \mid D(0, r)$ is trivial. We can choose $\rho \in(\delta, r)$ such that for $\delta<|z| \leq \rho, \eta Y(z) \subset K^{\varepsilon}(z)$, and so $L^{\varepsilon, r}(z)=K^{\varepsilon}(z)$ for $|z|>\rho$. Clearly, $K^{\varepsilon}$, and so $L^{\varepsilon, r}$, are locally trivial for $|z|>\rho$. Finally, $L^{\varepsilon, r}(z)=K^{\varepsilon}(z) \cup \eta Y(z)$ for $\frac{1}{2} \delta<|z|<r$. By the above comments, both $K^{\varepsilon}$ and $\eta Y(\cdot)$ are locally trivial on $\left\{\frac{1}{2} \delta<|z|<r\right\}$, and so is $L^{\varepsilon, r}$. We conclude that $z \rightarrow L^{\varepsilon, r}(z): \mathbb{C} \rightarrow 2^{\mathbb{C}}$ is a locally trivial analytic multifunction. 
Denote now by $L_{n}: \mathbb{C} \rightarrow 2^{\mathbb{C}}$ the multifunction $L^{\varepsilon, r}$, constructed above for $\varepsilon=2^{-n}$, and $r=r_{n}, \eta=\eta_{n}$ arbitrary, provided $r<\varepsilon^{2}, \eta R(r, \varepsilon)<\varepsilon$. Clearly, $L_{0}(z)=\bigcap_{n} L_{n}(z), L_{n}(z) \supset L_{n+1}(z), z \in \mathbb{C}, n=1,2, \ldots \quad$ Q.E.D.

\section{LIMITS OF LOCALLY TRIVIAL ANALYTIC MULTIFUNCTIONS}

Proposition 3.1. (a) If $K_{j}: G \rightarrow 2^{\mathbb{C}^{n}}, j=1, \ldots, s$ are locally trivial analytic multifunctions (have $(M A)$ property; cf. Terminology 1.5), then $K(z)=K_{1}(z) \cup$ $\cdots \cup K_{s}(z)$ is a locally trivial analytic multifunction (has $(M A)$ property).

(b) If $K: G \rightarrow 2^{\mathbb{C}^{n}}, G \subset \mathbb{C}^{k}$, is a locally trivial analytic multifunction (has $(M A)$ property) and $f: G^{*} \rightarrow G$ is an analytic mapping, then $z \rightarrow K(f(z)): G^{*} \rightarrow 2^{\mathbb{C}^{n}}$ is a locally trivial analytic multifunction (has $(M A)$ property).

(c) If $K$ is an in (b) and $(z, w) \rightarrow F_{z}(w)$ is a $\mathbb{C}^{m}$-valued analytic map defined near $\operatorname{gr}(K)$, then $z \rightarrow F_{z}(K(z)): G \rightarrow 2^{\mathbb{C}^{m}}$ is a locally trivial analytic multifunction (has $(M A)$ property).

(d) If $(z, \xi) \rightarrow K(z, \xi): G \times G^{*} \rightarrow 2^{\mathbb{C}^{n}}$ is a locally trivial analytic multifunction (has $(M A)$ property), and $F \subset G^{*}$ is compact, then $z \rightarrow \bigcup_{\xi \in F} K(z, \xi): G \rightarrow$ $2^{\mathrm{C}^{n}}$ is a locally trivial analytic multifunction (has (MA) property).

Proof. It is obvious that these operations preserve the class of trivial analytic multifunctions and commute with localization (restriction) and limits of decreasing sequences (as in Terminology 1.5). Q.E.D.

Lemma 3.2. Let $z \rightarrow L(z): G \rightarrow 2^{\mathbb{C}^{n}}$ be an usc compact-valued multifunction. Assume that for every $a \in G$ there is a multifunction $L_{a}: B(a) \rightarrow 2^{\mathrm{C}^{n}}$ with (MA) property, such that $\operatorname{gr}\left(L_{a} \mid B(a) \backslash\{a\}\right) \subset \operatorname{Int} \operatorname{gr}(L \mid B(a) \backslash\{a\})$. Then, for every neighborhood $W$ of $\operatorname{gr}(L)$ there is a locally trivial analytic multifunction $\widetilde{L}: G \rightarrow 2^{\mathbb{C}^{n}}$ such that $L(z) \subset \operatorname{Int} \widetilde{L}(z)$, for $z \in G$, and $\operatorname{gr} \widetilde{L} \subset W$.

Proof. We can assume that $B(a)$ is an Euclidean ball $B(a, r)$ with $r<$ $\operatorname{dist}(a, \partial G)$, and $L_{a}(z)$ is defined in a neighborhood of $\overline{B(a)}$. By the (MA) property, there is a locally trivial analytic multifunction $L_{n}$, defined near $\overline{B(a)}$, such that $\operatorname{gr} L_{n} \mid \partial B(a) \subset \operatorname{Int}(L \mid \partial B(a)), L(a) \subset L_{n}(a)$, and $\operatorname{gr} L_{n} \mid \overline{B(a)} \subset W$. Hence, there is $\varepsilon>0$, such that $\left\{(\zeta, w): \zeta \in \partial B(a)\right.$, $\left.\operatorname{dist}\left(w, L_{n}(\zeta)\right) \leq \varepsilon\right\} \subset$ $\operatorname{Int}(L \mid \partial B(a))$ and $\left\{(z, w): z \in \overline{B(a)}\right.$, $\left.\operatorname{dist}\left(w, L_{n}(z)\right) \leq \varepsilon\right\} \subset W$. By the continuity of $z \rightarrow L_{n}(z)$, the set $\left\{(z, w): z \in B(a)\right.$, dist $\left.\left(w, L_{n}(\zeta)\right)<\varepsilon\right\}$ is open, and so it contains $\{z\} \times L_{a}(z)$ for $z$ in some open neighborhood $H(a)$ of $a$, $H(a) \subset G$.

Let $L_{a}^{*}(z)=\left\{w \in \mathbb{C}^{n}: \operatorname{dist}\left(w, L_{n}(z)\right) \leq \varepsilon\right\}$ for $z \in \overline{B(a)}$. Clearly, $L_{a}^{*}: \overline{B(a)}$ $\rightarrow 2^{C^{n}}$ is compact-valued and usc, and $L_{a}^{*} \mid B(a)$ is a locally trivial analytic multifunction. By our construction,

$$
\begin{aligned}
& L(z) \subset L_{a}^{*}(z), \quad z \in H(a), \\
& \operatorname{gr}\left(L_{a}^{*} \mid \partial B(a)\right) \subset \operatorname{gr} L, \quad \operatorname{gr} L_{a}^{*} \subset W .
\end{aligned}
$$


Seeing that $H(a) \subset B(a)$ and $\bigcup_{a} H(a)=G$, we can select a countable covering $\{H(a(n))\}_{n=1}^{\infty}$ from $\{H(a)\}$, so that $\left.\{\overline{B(a(n)})\right\}_{n=1}^{\infty}$ is a locally finite family of compact subsets of $G$. The argument is identical with that in [11, Proof of Lemma 4.7]. Hence, the compact sets $\operatorname{gr}\left(L_{a(n)}^{*} \overline{B(a(n))}\right), n=1,2 \ldots$, , form a locally finite family relative to $G \times \mathbb{C}^{n}$, and so their union, which contains $\operatorname{gr} L$, by (3.1) is the graph of an usc, compact-valued multifunction, say $\widetilde{L}: G \rightarrow 2^{\mathrm{C}^{n}}$, such that $L(z) \subset \widetilde{L}(z), z \in G$. By (3.2), gr $\tilde{L} \subset W$.

Finally, $\widetilde{L}$ is a locally trivial analytic multifunction. Indeed, for $z_{0} \in G$ let $M=\left\{n: z_{0} \in B(a(n))\right\}$. Then, $M$ is a finite set and $H=\bigcap_{n \in M} H(a(n))$ is open. By (3.2), $\widetilde{L}(z)=\bigcup_{n \in M} L_{a(n)}^{*}(z)$, for $z \in H$. By Proposition 3.1(a), $\widetilde{L} \mid H$ is locally trivial. Q.E.D.

\section{APPLICATION OF $q$-PLURISUbHARMONIC FUNCTIONS}

Lemma 4.1. Let $\varphi(z, w)$ be a continuous $(n-1)$-plurisubharmonic function in an $(n-1)$-pseudoconvex open set $U \subset \mathbb{C}^{k} \times \mathbb{C}^{n}$ (cf. [7, Definition 4.1]). Fix $\varepsilon>0, \chi>0, \delta>0$. Then, there is a continuous function $u: U \rightarrow R$, such that

$$
\varphi(x)+\varepsilon|x|^{2}<u(x)<\varphi(x)+(\varepsilon+\chi)|x|^{2}+\delta, \quad x \in U,
$$

and for every $p \in U$, there is a ball $B(p)=\{|x-p|<r\}$, with $r<\operatorname{dist}(p, \partial u)$, and a continuous function $u_{p}(x)$ on $\overline{B(p)}$ with the following properties.

(i) If $p=(a, b) \in U$, then $u_{p}=u$ on $\overline{B(p)} \cap\{a\} \times \mathbb{C}^{n}$, while $u_{p}<u$ on $B(p) \backslash\{a\} \times \mathbb{C}^{n}$.

(ii) Every $u_{p}, p \in U$, has the representation $u_{p}(x)=\max \left(f_{1}(x), \ldots\right.$, $\left.f_{n}(x)\right), x \in \overline{B(p)}, m=m(p)$, where each $f_{j}$ is of the form

$$
f(x)=\min \left(\operatorname{Re} l_{1}(x), \ldots, \operatorname{Re} l_{n-1}(x), \operatorname{Re} h(x)\right), \quad x \in \mathbb{C}^{k+n},
$$

where $l_{1}(z, w), \ldots, l_{n-1}(z, w)$ are $\mathbb{C}$-affine functions and $h(z, w)$ is a seconddegree polynomial which satisfy the next condition:

(*) for every set of constants $\eta_{1}, \eta_{2}, \ldots, \eta_{n}$, and for every $z \in \mathbb{C}^{k}$, the systems of equations

$$
l_{i}(z, w)=\eta_{i}, \quad i=1,2, \ldots, n-1, \quad h(z, w)=\eta_{n},
$$

has at most two solutions $w$.

Proof (Sketch). This part of the argument relies heavily on the results and terminology of [11, 44 . Denote by $\widetilde{F}$ the class of all functions of the form (4.2) with $h(z, w)$ polynomial of degree two and $l_{1}(z, w), \ldots, l_{n-1}(z, w)$ complex affine functions. Denote by $F$ the subset of those $f$ in $\tilde{F}$ for which functions $l_{1}, \ldots, l_{n-1}, h$ satisfy condition $(*)$.

Assertion 1. $\widetilde{F}=\bar{F}$, the closure of $F$ in the uniform convergence on compact sets. 
Assertion 2. $F^{d}$, the dual class of functions to $F$ in the sense of [10, Definition $1.11]$ is equal to the class of all $(n-1)$-plurisubharmonic functions on $\mathbb{C}^{k+n}$.

The proof of Assertion 1 is elementary. The proof of Assertion 2 is similar to those of [11, Remark 5.1 and Theorem 5.8] and is omitted. The assertions mean that $F$ and the class $P$ consisting of all $(n-1)$-plurisubharmonic functions on $C^{k+n}$ satisfy all the assumptions of [11, Lemma 4.3 and Corollary 4.8]. Applying the last fact to the continuous function $\varphi$, we obtain that there is a continuous (and (n-1)-plurisubharmonic) function $v$ on $U$, such that $\varphi(x)+\varepsilon|x|^{2}<$ $v(x)<\varphi(x)+\varepsilon|x|^{2}+\delta, x \in U$, such that for every $p \in U$ there is a ball $\overline{B(p)} \subset$ $U$ such that $v \mid B(p)=\max \left(f_{1_{2}}, \ldots, f_{m}\right), m=m(x)$, where $f_{1}, \ldots, f_{m(x)} \in$ $F$. Define $u(z, w)=v(z, w)+\chi|z|^{2},(z, w) \in U$, and, for $p=(a, b) \in U$ and $(z, w) \in B(p)$, let $u_{p}(z, w)=v(z, w)+\chi|z|^{2}-\chi|z-a|^{2}$. Clearly, condition (i) holds. Since $u_{p}(z, w)=v(z, w)+\operatorname{Re} l(z),(z, w) \in B(p)$, where $l(z)=2 \chi a^{t} z-\chi|a|^{2}$, we get that $u_{p}=\max \left(f_{1}+\operatorname{Re} l, \ldots, f_{m}+\operatorname{Re} l\right)$ in $B(p)$. Since $l$ depends only on $z$, the representation (4.2) for $f_{j}+\operatorname{Re} l$ still satisfies (*), and so condition (ii) holds. Q.E.D.

\section{Proof of Theorem 1.3}

Outline of proof. The function $x \rightarrow-\log \operatorname{dist}(x, \operatorname{gr} K)$ is $(n-1)$-plurisubharmonic near $\operatorname{gr}(K)$ and, by lemma 4.1 , it can be approximated by a special $(n-1)$-plurisubharmonic function $u(z, w)$. The fact that this $u$ is made up from simple polynomials $l_{1}, \ldots, l_{n-1}, h$ satisfying conditions (i) and (ii) of Lemma 4.1, will allow us to approximate the sets $\operatorname{gr} K \cup\{u \geq C\}$ by locally trivial analytic multifunctions which are constructed, with use of Lemma 3.2, from simple multifunctions with (MA) property. The latter are produced from $l_{1}, \ldots, l_{n-1}, h$ as in the next lemma.

Lemma 5.1. Let $l_{1}, \ldots, l_{n-1}, h$ satisfy condition (ii) of Lemma 4.1 and $F \subset \mathbb{C}^{n}$ be compact. Let $K(z)=\left\{w \in \mathbb{C}^{n}:\left(l_{1}(z, w), \ldots, l_{n-1}(z, w), h(z, w)\right) \in F\right\}$. Then, the multifunction $z \rightarrow K(z): \mathbb{C}^{k} \rightarrow 2^{\mathrm{C}^{n}}$ has the $(M A)$ property.

Proof of Theorem 1.3.

Assertion. Let $U_{1}$ be a neighborhood of $\operatorname{gr}(K)$ in $G \times \mathbb{C}^{n}$ and $G^{*} \subset G$ be bounded and open. Let $U=U_{1} \backslash \operatorname{gr}(K)$. Assume that $u: U \rightarrow R$ satisfies conditions (i) and (ii) of Lemma 4.1, and $\lim _{x \rightarrow x_{0}} u(x)=+\infty$ for $x_{0} \in \partial(\operatorname{gr} K)$, $x \in U$. Assume that $C$ is such that the $\operatorname{set} X=(\{x \in U: u(x) \geq C\} \cup$ gr $K) \cap G^{*} \times \mathbb{C}^{n}$ is bounded and relatively closed in $G^{*} \times \mathbb{C}^{n}$. Then, for every neighborhood $W$ of $X$ there is a locally trivial analytic multifunction $z \rightarrow$ $\widetilde{L}(z): G^{*} \rightarrow 2^{\mathbb{C}^{n}}$, such that $X \subset \operatorname{gr}(\tilde{L}) \subset \mathbf{W}$.

We show first, how this implies Theorem 1.3. Since gr $K$ has the local maximum property of order $(k-1)$ (cf. [7, Definition 2.1]), its complement in $G \times \mathbb{C}^{n}$ is $(n-1)$-pseudoconvex relative to $G \times \mathbb{C}^{n}$, by [7, Theorem 4.2]. In other words (cf. [7, Definition 4.1]), there is an open set $U_{1}$ with $\operatorname{gr} K \subset U_{1} \subset G \times \mathbb{C}^{n}$, 
such that the function $\varphi(x)=-\log \operatorname{dist}(x, \operatorname{gr} K)$ is $(n-1)$-plurisubharmonic in the set $U=U_{1} \backslash \operatorname{gr}(K)$. Since $\varphi$ is continuous, by Lemma 4.1 there is a function $u: U \rightarrow R$, such that

$$
\varphi(x)+\frac{1}{2}|x|^{2}<u(x)<\varphi(x)+|x|^{2}+1, \quad x \in U,
$$

and $u(z, w)$ satisfies conditions (i), and (ii) of Lemma 4.1.

Choose a countable monotone covering $\left\{G_{s}\right\}_{s=1}$ of $G$, such that $\bar{G}_{s} \subset G_{s+1}$ and $\bar{G}_{s}$ are compact. We can assume without loss of generality that $\left(G_{s} \times \mathbb{C}^{n}\right) \subset$ $G_{s} \times B\left(0, R_{s}\right), R_{s}<+\infty$. By (5.1), we get

$$
\text { if } u(x) \geq C, \text { then } \operatorname{dist}(x, \operatorname{gr} K) \leq e^{-C} e^{|x|^{2}+1} .
$$

Now, for $x \in\left(G_{s} \times \mathbb{C}^{n}\right) \cap U$ the term $|x|^{2}$ is uniformly bounded, Hence, we can choose a sequence $C_{s} \nearrow+\infty$, such that for every $s=1,2 \ldots$, the set $X_{s}=\operatorname{gr}\left(K \mid G_{s}\right) \cup\left\{(z, w) \in U: z \in G_{s}, u(z, w) \geq C_{s}\right\}$ is closed in $G_{s} \times \mathbb{C}^{n}$. (Note that, by $(5.1), \lim u(x)=+\infty$ as $U \ni x \rightarrow x_{0} \in \operatorname{gr} K$.) By the same reason

$$
\bigcap_{r \geq s} X_{r} \cap G_{s} \times \mathbb{C}^{n}=\operatorname{gr} K \mid G_{s}, \quad s \geq 1
$$

Denote $W_{s}=\operatorname{gr}(K) \cup\left\{(z, w) \in U: u(z, w)>C_{s}\right\}, s=1,2, \ldots$ and $W_{0}=U$. Then,

$$
W_{s} \cap\left(G_{s} \times \mathbb{C}^{n}\right) \subset X_{s} \subset W_{s-1}, \quad s \geq 1 .
$$

By the assertion, for every $s \geq 1$, there is a locally trivial analytic multifunction $K_{s}: G_{s} \rightarrow 2^{\mathbb{C}^{n}}$, such that $X_{s} \subset \operatorname{gr}\left(K_{s}\right) \subset W_{s-1}, s \geq 1$. This and (5.4) imply that $K_{r+1}(z) \subset K_{r}(z)$, for $z \in G_{s}$ and $r \geq s$. By (5.3) and (5.4), $\bigcap_{r \geq s} K_{r}(z)=$ $K(z), z \in G_{s}$, as required.

Concerning the assertion, note first that $X$ is the graph of some compactvalued multifunction $L: G^{*} \rightarrow 2^{\mathbb{C}^{n}}$. We will prove the assertion by applying Lemma 3.2 to $L$; that is for each $a \in G^{*}$ we will construct a neighborhood $B(a)$ and a minorant multifunction $L_{a}: B(a) \rightarrow 2^{\mathbb{C}^{n}}$ satisfying assumptions of Lemma 3.2.

Consider an arbitrary $b \in\{w \in L(a): u(a, w)=C\}$. Denote $p=(a, b)$, and let $B=B(p)$ be a ball and $u_{p}=\max \left(f_{1}, \ldots, f_{m}\right)$ be a function in $B$, satisfying conditions (i) and (ii) of Lemma 4.1. We can assume without loss of generality that $B \cap \mathrm{gr} K=\varnothing$ and $B \subset U$. Clearly,

$$
\left\{u_{p} \geq C\right\} \cap B=\bigcap_{j=1}^{m}\left\{f_{j} \geq C\right\} \cap B .
$$

In turn, if $f_{j}=\min \left(\operatorname{Re} l_{1}, \ldots, \operatorname{Re} l_{n-1}, \operatorname{Re} h\right)$ as in Lemma 4.1(ii), then $\left\{f_{j} \geq\right.$ $\left.\left.C\} \cap B=B \cap \bigcap_{i=1}^{n-1}\left\{\operatorname{Re} l_{i} \geq C\right\} \cap\right\} \operatorname{Re} h \geq C\right\}$. (We suppress in the notation the dependence of $l_{i}$, and $h$ on $j$ and $b$.) We will now select a part of the latter set which is the graph of an analytic multifunction $L^{p, j}: B_{p, j} \rightarrow 2^{\mathbb{C}^{n}}$, 
where $a \in B_{p, j} \subset G^{*}$. (From these $L_{p, j}$ 's the minorant $L_{a}$ will be eventually constructed.) We distinguish two cases.

Case 1. The system of equations

$$
l_{1}(a, w)=l_{1}(a, b), \ldots, l_{n-1}(a, w)=l_{n-1}(a, b), \quad h(a, w)=h(a, b)
$$

has two distinct solutions in $w$, namely $b$ and $b^{*}$. Clearly, there exist a neighborhood $B_{p, j}$ of $a$ and disjoint neighborhoods $V$ and $V^{*}$ of $b$ and $b^{*}$, respectively, and a compact neighborhood $F_{0}$ of $b$, such that $F_{0} \subset V$, $B_{p, j} \times\left(V \cup V^{*}\right) \subset B_{p}$ and for every $\beta \in F_{0}$ and every $z \in B_{p, j}$ the system

$$
l_{1}(z, w)=l_{1}(a, \beta), \ldots, l_{n-1}(z, w)=l_{n-1}(a, \beta), \quad h(z, w)=h(a, \beta)
$$

has exactly two solutions $w$, one in $V$ and one in $V^{*}$.

Case 2. The system (5.6) has only one solution $w=b$. Then, there exist: a neighborhood $B_{p, j}$ of $a$, a compact neighborhood $F_{0}$ of $b$ and an open neighborhood $V$ of $b$, such that $F_{0} \subset V$ and $B_{p, j} \times V \subset B_{p}$, and for every $\beta \in F_{0}$ and for every $z \in B_{p, j}$ all solutions $w$ of the system (5.7) belong to $V$. For conformity of notation with the Case 1 , we let $V^{*}=\varnothing$ in Case 2.

In either case, we denote $F=F_{0} \cap\left\{w \in \mathbb{C}^{n}: f_{j}(a, w) \geq C\right\}$. For $z \in B_{p, j}$ let $L^{p, j}(z)$ to be the set of all solutions $w$ of (5.7) corresponding to $\beta \in F$. Since $F$ is compact, the construction implies easily that $z \rightarrow L^{p, j}(z): B_{p, j} \rightarrow 2^{\mathrm{C}^{n}}$ is an usc compact-valued multifunction. In Case 1 , it is obvious that $L^{p, j}$ is a trivial analytic multifunction. In Case 2 , multifunction $L^{p, j}(\cdot)$ has (MA) property by Lemma 5.1 .

With still fixed $p=(a, b)$, define now $L^{p}(z)=\bigcup_{j=1}^{m(p)} L^{p, j}(z)$ for $z \in B_{p}=$ $\bigcap_{j=1}^{m(p)} B_{p, j}$. By Proposition 3.1(a), $z \rightarrow L^{p}(z): B_{p} \rightarrow 2^{\mathbb{C}^{n}}$ has the (MA) property. By (5.5), the section $L^{p}(a)$ is a neighborhood of $b$ relative to $L(a)$. Since $b$ varies through the compact set $\{w: u(a, w)=C\}$, we can choose a finite family $\left\{L^{p}(a): p \in R\right\}$, where $R=\left\{\left(a, b_{1}\right), \ldots,\left(a, b_{r}\right)\right\}$, which covers the set $\left\{w \in \mathbb{C}^{n}: C \leq u(a, w)<C+\varepsilon\right\}$, for some $\varepsilon>0$. Denote $F^{*}=K(a) \cup\{w \in$ $\left.\mathbb{C}^{n}: C+\frac{1}{2} \varepsilon \leq u(a, w)\right\}$; clearly, $F^{*}$ is compact and there is a neighborhood $B^{*}$ of $a$ in $\mathbb{C}^{k}$, such that $F^{*} \subset K(z) \cup\left\{w \in \mathbb{C}^{n}: u_{p}(z, w)>C\right\}$ for every $z \in B^{*}$. Let, finally, $B(a)=B^{*} \cap \bigcap_{p \in R} B(p)$ and $L_{a}(z)=F^{*} \cup \bigcup_{p \in R} L^{p}(z)$, $z \in B(a)$. By our construction, $L_{a}(a)=L(a)$. By Proposition 3.1(a), the usc multifunction $z \rightarrow L_{a}(z): B(a) \rightarrow 2^{C^{n}}$ has (MA) property. Finally, gr $L_{a} \subset$ $\operatorname{gr} K \mid B(a) \cup\left\{(z, w): u_{p}(z, w) \geq C\right\}$. Since $u_{p}(z, w)<u(z, w)$ for $z \neq a$ (cf. Lemma 4.1(i)), we get $\operatorname{gr} L_{a} \mid B(a) \backslash\{a\} \subset \operatorname{Int}(\operatorname{gr} L \mid B(a) \backslash\{a\})$, as required in Lemma 3.2, which completes the proof of the assertion. Q.E.D.

Proof of Lemma 5.1 (Sketch). The assumptions on $l_{1}, l_{2}, \ldots, l_{n-1}, h$ imply that there is an additional $\mathbb{C}$-affine function $l(z, w)$, such that $\left(z_{1}, z_{2}, \ldots\right.$, $\left.z_{k}, \xi_{1}, \ldots, \xi_{n-1}, \xi\right)$ with $\xi_{j}=l_{j}(z, w), \zeta=l(z, w)$ form a biholomorphically equivalent coordinate system on $\mathbb{C}^{k} \times \mathbb{C}^{n}$. If the representation of the 
polynomial $h(z, w)$ in the new coordinates is $h_{0} \zeta^{2}+2 \zeta h_{1}\left(z, \xi^{\prime}\right)+h_{2}\left(z, \xi^{\prime}\right)$, where $\xi^{\prime}=\left(\xi_{1}, \ldots, \xi_{n-1}\right)$, then the solution to the system

$$
\left(l_{1}(z, w), \ldots, l_{n-1}(z, w), h(z, w)\right)=\left(\eta_{1}, \ldots, \eta_{n}\right)
$$

has the representation $\xi_{1}=\eta_{1}, \ldots, \xi_{n-1}=\eta_{n-1}$, and the set of values of $\zeta$ is $L(z, \eta)=-h_{0}^{-1} h_{1}\left(z, \eta^{\prime}\right)+L_{0}(\Delta(z, \eta))$, with $\Delta(z, \eta)=h_{0}^{-2} h_{1}\left(z, \eta^{\prime}\right)^{2}-$ $h_{0}^{-1}\left(h_{2}\left(z, \eta^{\prime}\right)-\eta_{n}\right)$, where $L_{0}$ is the multifunction with (MA) property from Corollary 2.2. Applying Corollary 2.2 and Proposition 3.1(b) and (c), we obtain that the multifunction $L: \mathbb{C}^{2 n} \rightarrow 2^{\mathbb{C}}$ has (MA) property. Returning to original coordinates, which amounts to applying Proposition 3.1(b) and (c) once again, we get that $K^{\prime}: C^{2 n} \rightarrow 2^{\mathbb{C}^{n}}$ has (MA) property, where $K^{\prime}(z, \eta)=$ the set of $w$ satisfying (5.8). Hence, $K(z)=\bigcup_{\eta \in F} K^{\prime}(z, \eta)$ has (MA) property by Proposition 3.2(d). Q.E.D.

\section{CONCLUDING REMARKS}

A. Further properties of analytic multifunctions. With Theorem 1.3 proven, we can conclude now that properties (a), (b), (c), and (d) of Proposition 3.1 hold for arbitrary analytic multifunctions. In the same way, we can easily obtain the following properties (starting with the obvious observation that they are satisfied by trivial analytic multifunctions).

(a) If $K: G \rightarrow 2^{\mathrm{C}^{n}}$ and $L: G \rightarrow 2^{\mathrm{C}^{m}}$ are analytic multifunctions, then $z \rightarrow$ $K(z) \times L(z): G \rightarrow 2^{\mathrm{C}^{m+n}}$ is analytic multifunction.

(b) If $K: G \rightarrow 2^{H}$, and $L: H \rightarrow 2^{\mathrm{C}^{s}}$ are analytic multifunctions, then so is their composition $L \circ H: G \rightarrow 2^{\mathbb{C}^{s}}$, where $(L \circ K(z)=\bigcup\{L(w): w \in K(z)\}$.

(c) If $K: G \rightarrow 2^{\mathrm{C}^{n}}$ is an analytic multifunction, then $\left.z \rightarrow \operatorname{co}(z)\right): G \rightarrow 2^{\mathrm{C}^{n}}$ is an analytic multifunction, where co $=$ the convex hull.

(d) If $\psi(z, w)$ is an $r$-plurisubharmonic function, $r \geq 0$, and $K: G \rightarrow 2^{\mathrm{C}^{n}}$ is an analytic multifunction, then $\varphi(z)=\max \{\psi(z, w): w \in K(z)\}$ is $r$ plurisubharmonic on the set $\{z:\{z\} \times K(z) \subset \operatorname{Dom} \psi\}$.

(e) If $K: G \rightarrow 2^{\mathrm{C}^{n}}$ is an analytic multifunction, then

$$
\varphi(z, w)=-\log \operatorname{dist}(w, K(z))
$$

is an $(n-1)$-plurisubharmonic function in $G \times \mathbb{C}^{n} \backslash \operatorname{gr}(K)$.

Remark. The above properties can be also obtained by applying results on $k$ maximum sets, cf. [7].

B. Proof of Remark 1.4 (Sketch). The method is essentially the same as in the case of Theorem 1.3, so we only indicate the necessary modifications. The assertion remains unchanged; it is, however, applied now to function $\varphi(z, w)$ as defined in property (e) above, because $\varphi(z, w)$ is continuous, due to the continuity of $z \rightarrow K(z)$. This and the uniform boundedness of $K(z)$ allows us to use $G_{s}=G, s=1,2, \ldots$ in the proof of Theorem 1.3. Q.E.D. 
C. The use of Forstneric result [2] in the proof of Lemma 2.1 shortens the argument but is not essential. One can also apply the theorem of Alexander and Wermer [1] and the author [8] about hulls with convex fibers to the image of the set $Y$ under the map $(z, w) \rightarrow\left(z, w^{-1}\right)$ (plus the zero section $\left.D(0, r) \times\{0\}\right)$, which has, indeed, convex vertical sections. (Some additional argument is still needed.)

\section{REFERENCES}

1. H. Alexander and J. Wermer, Polynomial hulls with convex fibers, Math. Ann. 271 (1985), 99-109.

2. F. Forstneric, Polynomial hulls of sets fibered over the circle, Indiana Univ. Math. J. (to appear).

3. K. Oka, Note sur les families de fonctions analytiques multiformes, etc, J. Sci. Hiroshima Univ. Ser. A 4 (1934), 93-98.

4. Z. Slodkowski, Analytic set-valued functions and spectra, Math. Ann. 256 (1981), 363-386.

5. _ A criterion for subharmonicity of a function of the spectrum, Studia Math. 75 (1982), $37-49$.

6. __, Analytic multifunctions, q-plurisubharmonic functions and uniform algebras, Proc. Conf. Banach Algebras and Several Complex Variables (F. Greenleaf and D. Gulick, eds.), Contemp. Math., vol. 32, Amer. Math. Soc. Providence, R.I., 1984, pp . 243-258.

7. _. Local maximum property and q-plurisubharmonic functions in uniform algebras, J. Math. Anal. Appl. 115 (1986), 105-130.

8. _ Polynomials hulls with convex sections and interpolating spaces, Proc. Amer. Math. Soc. 96 (1986), 255-260.

9. __ An analytic set-valued selection and its applications to the corona theorem, to polynomial hulls and joint spectra, Trans. Amer. Math. Soc. 294 (1986), 367-377.

10. _ Pseudoconvex classes of functions. I. Pseudoconcave and pseudoconvex sets, Pacific J. Math. 134 (1988), 343-376.

11. _ Pseudoconvex classes of functions. II. Affine pseudoconvex classes on $R^{\eta}$ Pacific J. Math. (to appear).

12. K. Oka, Unramified domains without points at infinity, Collected Papers, Springer-Verlag, Berlin, 1984.

13. E. Vesentini, Caratheodory distances and Banach algebras, Adv. in Math. 47 (1983), 50-73.

14. H. Yamaguchi, Sur une uniformite' des surfaces constantes d'une fonction entie re de deux variables complexes, J. Math. Kyoto Univ. 13 (3) (1973), 417-433.

Note added July 17, 1988. The referee has pointed out that a result closely related to the case $k=n=1$ of Theorem 1.3 above was stated by E. Vesentini in [13], pp. 66 (bottom) and 67 (top). Vesentini asserts that, for dimensions $k=n=1$, decreasing approximations $K_{r}$ (like in Theorem 3.1) can be found so that graphs of multifunctions $K_{r}$ satisfy a certain condition $(L)$. E. Vesentini credits H. Yamaguchi $[14$, p. 420-421] with this theorem. However, according to Vesentini, a set $F \subset C^{2}$ has the property $(L)$ if it is covered (locally) by graphs of single-valued analytic functions, while Yamaguchi defines that $F$ has the property $(L)$ if it is covered by open pieces of nonsingular analytic varieties. If we are right in assuming that Yamaguchi bases himself on Oka [12, Lemma II, p. 171], only the latter version seems to be actually proved. Since nonsingular analytic variety in $C^{2}$ is locally given by one of the equations $w=f(z)$ or $z=g(w)$, the result is sufficient for Yamaguchi and Vesentini's applications (see [5] for a more general situation of this kind), but it does not directly imply the full strength of Theorem 1.3 of the present paper. 60680

Department of Mathematics, University of Illinois at Chicago, Chicago, Illinois 